\title{
On Metric Dimension of Two Constructed Families from Antiprism Graph
}

\author{
M. Ali ${ }^{1,2}$, G. Ali ${ }^{1,2}$ and M. T. Rahim ${ }^{2}$ \\ ${ }^{1}$ Centre for Mathematical Imaging Techniques and Dept. Math. Sciences, University of Liverpool, UK \\ ${ }^{2}$ Dept. of Mathematics, National University of Computer \& Emerging Sciences, Peshawar, Pakistan \\ Email:murtaza_psh@yahoo.com,tariq.rahim@nu.edu.pk,gohar.ali@nu.edu.pk
}

Received: 1 Jan. 2012, Revised: 13 May 2012, Accepted: 2 Jul. 2012

Published online: 1 Jan. 2013

\begin{abstract}
In this paper we compute the metric dimension of two families of graphs constructed from antiprism graph.
\end{abstract}

Keywords: Metrics dimension, basis, resolving set, antiprism

\section{Introduction}

For a connected graph $G$, the distance $d(u, v)$ between two vertices $u, v \in V(G)$ is the length of a shortest path between them in $G$. Let $W=\left\{w_{1}, w_{2}, \ldots, w_{k}\right\}$ be an ordered set of vertices of $G$ and let $v$ be a vertex of $G$, the representation of the vertex $v$ with respect to $W$, denoted by $r(v \mid W)$ is the $k$ tuple $\left(d\left(v, w_{1}\right), d\left(v, w_{2}\right), \ldots . ., d\left(v, w_{k}\right)\right)$. If distinct vertices of $G$ have distinct representations with respect to $W$, then $W$ is called a resolving set or locating set for $G$ [2]. A resolving set of minimum cardinality is called a metric basis for $G$ and this cardinality is the metric dimension of $G \operatorname{dim}(G)$,

For a given ordered set of vertices $W=\left\{w_{1}, w_{2}, \ldots ., w_{k}\right\}$ of a graph $G$, the ith component of $r(v \mid W)$ is 0 if and only if $v=w_{i}$. Thus, to show that $W$ is a resolving set it suffices to verify that $r(x \mid W) \neq r(y \mid W)$ for each pair of distinct vertices $x, y \in V(G) \backslash W$.

Motivated by the problem of uniquely determining the location of an intruder in a network, the concept of metric dimension was introduced by Slater in $[10,11]$ and studied independently by Harary and Melter in [3]. Applications of this invariant to the navigation of robots in networks are discussed in [8] and applications to chemistry in [2] while applications to problems of pattern recognition and image processing, some of which involve the use of hierarchical data structures are given in [9]. In $[4,5,6]$ Imran et al. proved the metric dimension of some families of convex polytopes.

In [2] Chartrand et al. proved that a graph has metric dimension 1 if and only if it is a path, hence paths on $n$ vertices constitute a family of graphs with constant metric dimension. Similarly, cycle with $n \geq 3$ vertices also constitute such a family of graphs as their metric dimension is 2. In [1] J. Caceres et al. proved that:

$$
\operatorname{dim}\left(p_{m} \times C_{n}\right)= \begin{cases}2, & \text { if } n, \text { is odd } \\ 3, & \text { other wise }\end{cases}
$$


Since prisms $D_{n}$ are the trivalent plane graphs obtained by the cartesian product of the path $P_{2}$ with a cycle $C_{n}$; they also constitute a family of 3 -regular graphs with constant metric dimension. Javaid et al. proved in [7] that the graph of antiprism $A_{n}$ constitutes a family of regular graphs with constant metric dimension and $\operatorname{dim}\left(A_{n}\right)=3$ for every $n \geq 5$.

In this paper, we extend this study by considering two families of graph which are constructed from antiprism.

The antiprism $A_{n}, n \geq 3$, consists of an outer $n$-cycle $a_{1} a_{2} \ldots a_{n}$, an inner $n$-cycle $b_{1} b_{2} \ldots b_{n}$, and a set of $n$ spokes $b_{i} a_{i}$ and $b_{i+1} a_{i}, i=1,2,3, \ldots, n$ where $n+i$ is taken modulo $n$.

The graph $H_{n}$ is constructed from the graph $A_{n}$ as follows: We delete the edges $a_{i} a_{i+1}$ from $A_{n}$. For each $i=1,2, \ldots, n$, we introduce new vertices $c_{i}$ and $d_{i}$ for $a_{i}$ and $b_{i}$ respectively. For each $i=1,2, \ldots, n$, introduce new edges $b_{i} c_{i}, a_{i} d_{i}, c_{i} d_{i}$ and $b_{i} c_{i}$, where $n+i$ is taken modulo $n$.

The graph $R_{n}$ is constructed from the graph $A_{n}$ as follows: We delete the edges $a_{i} a_{i+1}$ from $A_{n}$. For each $i=1,2, \ldots, n$, we introduce new vertices $c_{i}$ and $d_{i}$ for $a_{i}$ and $b_{i}$ respectively. For each $i=1,2, \ldots, n$ introduce new edges $b_{i} c_{i}, a_{i} d_{i}, c_{i} d_{i}, d_{i} d_{i+1}$ and $b_{i} c_{i}$ where $n+i$ is taken modulo $n$.

\section{Main Results}

Theorem: Let $n \geq 6$ be an integer then $\operatorname{dim}\left(H_{n}\right)=3$.

Proof. We distinguish two cases.

Case (i): $n=2 k, k \geq 3, k \in I N$. We consider $W=\left\{b_{1}, b_{2}, b_{k+1}\right\} \subset V\left(H_{n}\right)$. We show that $W$ is a resolving set for $V\left(H_{n}\right)$. For this we find the representations of the vertices of $V\left(H_{n} \wedge W\right.$ with respect to $W$. The representations of the vertices are as follows;

$$
\begin{aligned}
& r\left(b_{i} \mid W\right)= \begin{cases}(i-1, i-2,1+k-i), & \text { for } 3 \leq i \leq k ; \\
(2 k-i+1,2 k+2-i, i-1-k), & k+2 \leq i \leq n .\end{cases} \\
& r\left(c_{i} \mid W\right)= \begin{cases}(1,2, k+1), & \text { for } i=1 ; \\
(i, i-1, k+2-i), & \text { for } 2 \leq i \leq k+1 ; \\
(2 k+2-i, 3+2 k-i, i-k), & \text { for } k+2 \leq i \leq n .\end{cases} \\
& r\left(a_{i} \mid W\right)= \begin{cases}(1,1, k), & i=1 ; \\
(i, i-1, k+1-i), & 2 \leq i \leq k ; \\
(k, k, 1), & i=k+1 ; \\
(2 k+1-i, 2 k+2-i, i-k), & k+2 \leq i \leq n .\end{cases} \\
& r\left(d_{i} \mid W\right)= \begin{cases}(2,2, k+1), & i=1 ; \\
(i+1, i, k+2-i), & 2 \leq i \leq k ; \\
(k+1, k+1,2), & i=k+1 ; \\
(2 k+2-i, 2 k+3-i, i+1-k), & k+2 \leq i \leq n .\end{cases}
\end{aligned}
$$

Note that there are no two vertices having the same representations implying that $\operatorname{dim}\left(H_{n}\right) \leq 3$. 
Now we show that $\operatorname{dim}\left(H_{n}\right) \geq 3$, by proving that there is no resolving set $W^{\prime}$ with $\left|W^{\prime}\right|=2$. We have the following possibilities;

(1). Both vertices belong to $\left\{b_{i}: i=1,2, \ldots, n\right\} \subset V\left(H_{n}\right)$. Without loss of generality we suppose that one resolving vertex is $b_{1}$ and the other is $b_{t},(2 \leq t \leq k+1)$. For $2 \leq t \leq k$ we have $r\left(c_{1} \mid\left\{b_{1}, b_{t}\right\}\right)=r\left(a_{n} \mid\left\{b_{1}, b_{t}\right\}\right)=(1, t) \quad$ and $\quad$ for $\quad t=k+1$, we have $r\left(a_{k} \mid\left\{b_{1}, b_{t}\right\}\right)=r\left(a_{k+1} \mid\left\{b_{1}, b_{t}\right\}\right)=(k, 1)$, a contradiction.

(2). Both vertices belong to $\left\{c_{i}: i=1,2, \ldots, n\right\} \subset V\left(H_{n}\right)$. Without loss of generality we suppose that one resolving vertex is $c_{1}$, and the other is $c_{t},(2 \leq t \leq k+1)$. Then for $2 \leq t \leq k$ we have $r\left(b_{n} \mid\left\{c_{1}, c_{t}\right\}\right)=r\left(a_{n} \mid\left\{c_{1}, c_{t}\right\}\right)=(2, t+1)$ for $t=k+1$ is $r\left(a_{k} \mid\left\{c_{1}, c_{t}\right\}\right)=r\left(a_{k+1} \mid\left\{c_{1}, c_{t}\right\}\right)=(k+1,1)$, a contradiction.

(3). Both vertices belong to $\left\{a_{i}: i=1,2, \ldots, n\right\} \subset V\left(H_{n}\right)$. We suppose that one resolving vertex is $a_{1}$ and the other is $a_{t},(2 \leq t \leq k+1)$. Then for $2 \leq t \leq k$ we have $r\left(c_{1} \mid\left\{a_{1}, a_{t}\right\}\right)=r\left(b_{n} \mid\left\{a_{1}, a_{t}\right\}\right)=(2, t+1)$ and for $t=k+1$, is $r\left(b_{k+1} \mid\left\{a_{1}, a_{t}\right\}\right)=r\left(b_{k} \mid\left\{a_{1}, a_{t}\right\}\right)=(k, 1)$, a contradiction.

(4). Both vertices belong to $\left\{d_{i}: i=1,2, \ldots, n\right\} \subset V\left(H_{n}\right)$. We suppose that one resolving vertex is $d_{1}$ and the other is $d_{t},(2 \leq t \leq k+1)$. For $2 \leq t \leq k$ we have $r\left(a_{n} \mid\left\{d_{1}, d_{t}\right\}\right)=r\left(b_{n} \mid\left\{d_{1}, d_{t}\right\}\right)=(3, t+2)$ and for $t=k+1$, we have $r\left(c_{k+1} \mid\left\{d_{1}, d_{t}\right\}\right)=r\left(c_{k+1} \mid\left\{d_{1}, d_{t}\right\}\right)=(k+2,1)$, a contradiction.

(5). One vertex belong to $\left\{b_{i}: i=1,2, \ldots, n\right\} \subset V\left(H_{n}\right)$ and another vertex belong to $\left\{c_{i}: i=1,2, \ldots, n\right\} \subset V\left(H_{n}\right)$. Without loss of generality we suppose that one resolving vertex is $b_{1}$ and the other is $c_{t},(1 \leq t \leq k+1)$. For $1 \leq t \leq k$ we have $r\left(a_{n} \mid\left\{b_{1}, c_{t}\right\}\right)=r\left(b_{n} \mid\left\{b_{1}, c_{t}\right\}\right)=(1, t+1)$ and for $t=k+1$, is $r\left(a_{k} \mid\left\{b_{1}, c_{t}\right\}\right)=r\left(a_{k+1} \mid\left\{b_{1}, c_{t}\right\}\right)=(k, 2)$, a contradiction.

(6). One vertex belong to $\left\{b_{i}: i=1,2, \ldots, n\right\} \subset V\left(H_{n}\right)$ and another vertex belong to $\left\{a_{i}: i=1,2, \ldots, n\right\} \subset V\left(H_{n}\right)$. Without loss of generality we suppose that one resolving vertex is $b_{1}$ and the other is $a_{t},(1 \leq t \leq k+1)$. Then for $1 \leq t \leq k$ we have $r\left(a_{n} \mid\left\{b_{1}, a_{t}\right\}\right)=r\left(c_{1} \mid\left\{b_{1}, a_{t}\right\}\right)=(1, t+1)$ and for $t=k+1, r\left(b_{k} \mid\left\{b_{1}, a_{t}\right\}\right)=r\left(a_{k+2} \mid\left\{b_{1}, a_{t}\right\}\right)=(k-1,2)$, a contradiction.

(7). One vertex belong to $\left\{c_{i}: i=1,2, \ldots, n\right\} \subset V\left(H_{n}\right)$ and another vertex belong to $\left\{a_{i}: i=1,2, \ldots, n\right\} \subset V\left(H_{n}\right)$. Without loss of generality we suppose that one resolving vertex is $c_{1}$ and the other is $a_{t},(1 \leq t \leq k+1)$. For $1 \leq t \leq k-1$ we have $r\left(a_{n} \mid\left\{c_{1}, a_{t}\right\}\right)=r\left(b_{n} \mid\left\{c_{1}, a_{t}\right\}\right)=(2, t+1)$ and for $t=k, \quad r\left(a_{k+1} \mid\left\{c_{1}, a_{t}\right\}\right)=r\left(c_{k} \mid\left\{c_{1}, a_{t}\right\}\right)=(k, 2), \quad$ similarly $\quad$ for $\quad t=k+1$, we have $r\left(a_{k} \mid\left\{c_{1}, a_{t}\right\}\right)=r\left(c_{k+2} \mid\left\{c_{1}, a_{t}\right\}\right)=(k+1,2)$, a contradiction.

(8). One vertex belong to $\left\{a_{i}: i=1,2, \ldots, n\right\} \subset V\left(H_{n}\right)$ and another vertex belong to $\left\{d_{i}: i=1,2, \ldots, n\right\} \subset V\left(H_{n}\right)$. Without loss of generality we suppose that one resolving vertex is $a_{1}$ and the other is $d_{t},(1 \leq t \leq k+1)$. For $1 \leq t \leq k$ we have $r\left(a_{n} \mid\left\{a_{1}, d_{t}\right\}\right)=r\left(b_{n} \mid\left\{a_{1}, d_{t}\right\}\right)=(2, t+2)$ and for $t=k+1$ the representation is $r\left(c_{k+1} \mid\left\{a_{1}, d_{t}\right\}\right)=r\left(c_{k+2} \mid\left\{a_{1}, d_{t}\right\}\right)=(k+2,1)$, a contradiction.

(9). One vertex belong to $\left\{b_{i}: i=1,2, \ldots, n\right\} \subset V\left(H_{n}\right)$ and another vertex belong to $\left\{d_{i}: i=1,2, \ldots, n\right\} \subset V\left(H_{n}\right)$. Without loss of generality we suppose that one resolving vertex is $b_{1}$ and the other is $d_{t}, \quad(1 \leq t \leq k+1)$. For $1 \leq t \leq k-1 \quad$ we have $r\left(a_{n} \mid\left\{b_{1}, d_{t}\right\}\right)=r\left(b_{n} \mid\left\{b_{1}, d_{t}\right\}\right)=(1, t+2)$ and for $t=k$ the representation is 
$r\left(a_{k} \mid\left\{b_{1}, d_{t}\right\}\right)=r\left(c_{k} \mid\left\{b_{1}, d_{t}\right\}\right)=(k, 1), \quad$ similarly $\quad$ for $\quad t=k+1, \quad$ we have $r\left(a_{k+1} \mid\left\{b_{1}, d_{t}\right\}\right)=r\left(c_{k+2} \mid\left\{b_{1}, d_{t}\right\}\right)=(k, 1)$, a contradiction.

(10). One vertex belong to $\left\{c_{i}: i=1,2, \ldots, n\right\} \subset V\left(H_{n}\right)$ and another vertex belong to $\left\{d_{i}: i=1,2, \ldots, n\right\} \subset V\left(H_{n}\right)$. Without loss of generality we suppose that one resolving vertex is $c_{1}$ and the other is $d_{t}, \quad(1 \leq t \leq k+1)$. For $1 \leq t \leq k-1 \quad$ we have $r\left(a_{n} \mid\left\{c_{1}, d_{t}\right\}\right)=r\left(b_{n} \mid\left\{c_{1}, d_{t}\right\}\right)=(2, t+2)$ and for $t=k, r\left(a_{k} \mid\left\{c_{1}, d_{t}\right\}\right)=r\left(c_{k} \mid\left\{c_{1}, d_{t}\right\}\right)=(k+1,1)$, similarly for $t=k+1$, we have $r\left(a_{k+1} \mid\left\{c_{1}, d_{t}\right\}\right)=r\left(c_{k+2} \mid\left\{c_{1}, d_{t}\right\}\right)=(k+1,1)$, a contradiction.

Hence, from above it follows that there is no resolving set with two vertices for $V\left(H_{n}\right)$ implying that $\operatorname{dim}\left(H_{n}\right)=3$

Case(ii): $n=2 k+1, k \geq 3, k \in I N$. Let $W=\left\{b_{1}, b_{2}, b_{k+2}\right\} \subset V\left(H_{n}\right)$. We show that $W$ is a resolving set for $V\left(H_{n}\right)$. For this we find the representations of vertices of $V\left(H_{n}\right) \backslash W$ with respect to $W$.

The representations of the vertices are as follow;

$$
\begin{aligned}
& r\left(a_{i} \mid W\right)= \begin{cases}(1,1, k+1), & \text { for } i=1 ; \\
(i, i-1, k+2-i), & \text { for } 2 \leq i \leq k+1 ; \\
(2 k+2-i, 3+2 k-i, i-k-1), & \text { for } k+2 \leq i \leq n .\end{cases} \\
& r\left(b_{i} \mid W\right)= \begin{cases}(i-1, i-2,2+k-i), & \text { for } 3 \leq i \leq k+1 ; \\
(2 k-i+2,2 k+3-i, i-2-k), & k+3 \leq i \leq n .\end{cases} \\
& r\left(c_{i} \mid W\right)= \begin{cases}(1,2, k+1), & i=1 ; \\
(i, i-1, k+3-i), & 2 \leq i \leq k+1 ; \\
(k+1, k+1,1), & i=k+2 ; \\
(2 k+3-i, 2 k+4-i, i-1-k), & k+3 \leq i \leq n .\end{cases} \\
& r\left(d_{i} \mid W\right)= \begin{cases}(2,2, k+2), & \text { for } i=1 ; \\
(i+1, i, k+3-i), & \text { for } 2 \leq i \leq k+1 ; \\
(2 k+3-i, 4+2 k-i, i-k), & \text { for } k+2 \leq i \leq n .\end{cases}
\end{aligned}
$$

Proceeding on same line as in case(i) we note that there are no two vertices having the same representations, implying that $\operatorname{dim}\left(H_{n}\right) \leq 3$.

Also as in case(1), it can be shown that there is no set $W^{\prime}$ with $\left|W^{\prime}=2\right|$, such that $W^{\prime}$ is a resolving set for $V\left(H_{n}\right)$ for $n \geq 6$ and $n$ is odd. Thus, $\operatorname{dim}\left(H_{n}\right) \geq 3$. Hence $\operatorname{dim}\left(H_{n}\right)=3$ From case(i) and case(ii) we get $\operatorname{dim}\left(H_{n}\right)=3$.

Theorem: Let $n \geq 6$ be an integer then $\operatorname{dim}\left(R_{n}\right)=3$.

Proof. We distinguish two cases:

Case(i) $n=2 k, k \geq 3, k \in I N$. Suppose $W=\left\{b_{1}, b_{2}, b_{k+1}\right\} \subset V\left(R_{n}\right)$. We show that $W$ is a resolving set for $V\left(R_{n}\right)$. For this we find the representations of the vertices of $V\left(R_{n}\right) \backslash W$ with respect to $W$.

The representations of the vertices are as follows; 


$$
\begin{aligned}
& r\left(b_{i} \mid W\right)= \begin{cases}(i-1, i-2,1+k-i), & \text { for } 3 \leq i \leq k ; \\
(2 k-i+1,2 k+2-i, i-1-k), & k+2 \leq i \leq n .\end{cases} \\
& r\left(c_{i} \mid W\right)= \begin{cases}(1,2, k+1), & \text { for } i=1 ; \\
(i, i-1, k+2-i), & \text { for } 2 \leq i \leq k+1 ; \\
(2 k+2-i, 3+2 k-i, i-k), & \text { for } k+2 \leq i \leq n .\end{cases} \\
& r\left(a_{i} \mid W\right)= \begin{cases}(1,1, k), & i=1 ; \\
(i, i-1, k+1-i), & 2 \leq i \leq k ; \\
(k, k, 1), & i=k+1 ; \\
(2 k+1-i, 2 k+2-i, i-k), & k+2 \leq i \leq n .\end{cases} \\
& r\left(d_{i} \mid W\right)= \begin{cases}(2,2, k+1), & i=1 ; \\
(i+1, i, k+2-i), & 2 \leq i \leq k ; \\
(k+1, k+1,2), & i=k+1 ; \\
(2 k+2-i, 2 k+3-i, i+1-k), & k+2 \leq i \leq n .\end{cases}
\end{aligned}
$$

We note that there are no two vertices having the same representations implying that $\operatorname{dim}\left(R_{n}\right) \leq 3$.

Now we show that $\operatorname{dim}\left(R_{n}\right) \geq 3$, by proving that there is no resolving set $W^{\prime}$ with $\left|W^{\prime}\right|=2$. We have the following possibilities,

(1). Both vertices belong to $\left\{b_{i}: i=1,2, \ldots, n\right\} \subset V\left(R_{n}\right)$. Without loss of generality we suppose the resolving vertices $b_{1}$ and $b_{t},(2 \leq t \leq k+1)$. For $2 \leq t \leq k$ we have $r\left(c_{1} \mid\left\{b_{1}, b_{t}\right\}\right)=r\left(a_{n} \mid\left\{b_{1}, b_{t}\right\}\right)=(1, t)$ and for $t=k+1, r\left(a_{k} \mid\left\{b_{1}, b_{t}\right\}\right)=r\left(a_{k+1} \mid\left\{b_{1}, b_{t}\right\}\right)=(k, 1)$, a contradiction.

(2). Both vertices belong to $\left\{c_{i}: i=1,2, \ldots, n\right\} \subset V\left(R_{n}\right)$. We suppose that one resolving vertex is $c_{1}$ and the other is $c_{t},(2 \leq t \leq k+1)$. For $2 \leq t \leq k$ we have $r\left(b_{n} \mid\left\{c_{1}, c_{t}\right\}\right)=r\left(a_{n} \mid\left\{c_{1}, c_{t}\right\}\right)=(2, t+1)$ and for $t=k+1, r\left(b_{n} \mid\left\{c_{1}, c_{t}\right\}\right)=r\left(a_{n} \mid\left\{c_{1}, c_{t}\right\}\right)=(2, t+1)$, a contradiction.

(3). Both vertices belong to $\left\{a_{i}: i=1,2, \ldots, n\right\} \subset V\left(R_{n}\right)$. We suppose that one resolving vertex is $a_{t}$ and the other is $a_{t},(2 \leq t \leq k+1)$. For $2 \leq t \leq k$ we have $r\left(c_{1} \mid\left\{a_{1}, a_{t}\right\}\right)=r\left(b_{n} \mid\left\{a_{1}, a_{t}\right\}\right)=(2, t+1)$ and for $t=k+1 \quad$ we have $\quad r\left(b_{k+1} \mid\left\{a_{1}, a_{t}\right\}\right)=r\left(b_{k} \mid\left\{a_{1}, a_{t}\right\}\right)=(k, 1)$, a contradiction.

(4). Both vertices belong to $\left\{d_{i}: i=1,2, \ldots, n\right\} \subset V\left(R_{n}\right)$. We suppose that one resolving vertex is $d_{1}$ and the other is $d_{t},(2 \leq t \leq k+1)$. For $2 \leq t \leq k$ we have $r\left(a_{n} \mid\left\{d_{1}, d_{t}\right\}\right)=r\left(b_{n} \mid\left\{d_{1}, d_{t}\right\}\right)=(3, t+2)$ and for $t=k+1, r\left(c_{k+1} \mid\left\{d_{1}, d_{t}\right\}\right)=r\left(c_{k+1} \mid\left\{d_{1}, d_{t}\right\}\right)=(k+2,1)$, a contradiction.

(5). One vertex belong to $\left\{b_{i}: i=1,2, \ldots, n\right\} \subset V\left(R_{n}\right)$ and another belong to $\left\{c_{i}: i=1,2, \ldots, n\right\} \subset V\left(R_{n}\right)$. Without loss of generality we suppose that one resolving vertex is $b_{1}$ and the other is $c_{t},(1 \leq t \leq k+1)$. For $1 \leq t \leq k \quad$ we have $r\left(a_{n} \mid\left\{b_{1}, c_{t}\right\}\right)=r\left(b_{n} \mid\left\{b_{1}, c_{t}\right\}\right)=(1, t+1)$ and for $t=k+1$, $r\left(a_{k} \mid\left\{b_{1}, c_{t}\right\}\right)=r\left(a_{k+1} \mid\left\{b_{1}, c_{t}\right\}\right)=(k, 2)$, a contradiction.

(6). One vertex belong to $\left\{b_{i}: i=1,2, \ldots, n\right\} \subset V\left(R_{n}\right)$ and another belong to $\left\{a_{i}: i=1,2, \ldots, n\right\} \subset V\left(R_{n}\right)$. Without loss of generality we suppose that one resolving vertex is $b_{1}$ and the other is $a_{t},(1 \leq t \leq k+1)$. 
For $1 \leq t \leq k$ we have $r\left(a_{n} \mid\left\{b_{1}, a_{t}\right\}\right)=r\left(c_{1} \mid\left\{b_{1}, a_{t}\right\}\right)=(1, t+1) \quad$ and $\quad$ for $\quad t=k+1$ is $r\left(b_{k} \mid\left\{b_{1}, a_{t}\right\}\right)=r\left(a_{k+2} \mid\left\{b_{1}, a_{t}\right\}\right)=(k-1,2)$, a contradiction.

(7). One vertex belong to $\left\{c_{i}: i=1,2, \ldots, n\right\} \subset V\left(R_{n}\right)$ and another belong to $\left\{a_{i}: i=1,2, \ldots, n\right\} \subset V\left(R_{n}\right)$. Without loss of generality we suppose that one resolving vertex is $c_{1}$ and the other is $a_{t},(1 \leq t \leq k+1)$. For $1 \leq t \leq k-1 \quad$ we have $\quad r\left(a_{n} \mid\left\{c_{1}, a_{t}\right\}\right)=r\left(b_{n} \mid\left\{c_{1}, a_{t}\right\}\right)=(2, t+1) \quad$ and $\quad$ for $t=k$, $r\left(a_{k+1} \mid\left\{c_{1}, a_{t}\right\}\right)=r\left(c_{k} \mid\left\{c_{1}, a_{t}\right\}\right)=(k, 2), \quad$ similarly for $t=k+1 \quad$ we have $r\left(a_{k} \mid\left\{c_{1}, a_{t}\right\}\right)=r\left(c_{k+2} \mid\left\{c_{1}, a_{t}\right\}\right)=(k+1,2)$, a contradiction.

(8). One vertex belong to $\left\{a_{i}: i=1,2, \ldots, n\right\} \subset V\left(R_{n}\right)$ and another belong to $\left\{d_{i}: i=1,2, \ldots, n\right\} \subset V\left(R_{n}\right)$. Without loss of generality we suppose that one resolving vertex $a_{1}$ and the other is $d_{t},(1 \leq t \leq k+1)$. For $1 \leq t \leq k$ we have $r\left(a_{n} \mid\left\{a_{1}, d_{t}\right\}\right)=r\left(b_{n} \mid\left\{a_{1}, d_{t}\right\}\right)=(2, t+2) \quad$ and for $t=k+1$, is $r\left(c_{k+1} \mid\left\{a_{1}, d_{t}\right\}\right)=r\left(c_{k+2} \mid\left\{a_{1}, d_{t}\right\}\right)=(k+2,1)$, a contradiction.

(9). One vertex belong to $\left\{b_{i}: i=1,2, \ldots, n\right\} \subset V\left(R_{n}\right)$ and another belong to $\left\{d_{i}: i=1,2, \ldots, n\right\} \subset V\left(R_{n}\right)$. Without loss of generality we suppose that one resolving vertex is $b_{1}$ and the other is $d_{t},(1 \leq t \leq k+1)$. For $1 \leq t \leq k-1 \quad$ we have $r\left(a_{n} \mid\left\{b_{1}, d_{t}\right\}\right)=r\left(b_{n} \mid\left\{b_{1}, d_{t}\right\}\right)=(1, t+2)$ and for $t=k$ is $r\left(a_{k} \mid\left\{b_{1}, d_{t}\right\}\right)=r\left(c_{k} \mid\left\{b_{1}, d_{t}\right\}\right)=(k, 1)$, similarly for $t=k+1$ than we have $r\left(a_{k+1} \mid\left\{b_{1}, d_{t}\right\}\right)=r\left(c_{k+2} \mid\left\{b_{1}, d_{t}\right\}\right)=(k, 1)$, a contradiction.

(10). One vertex belong to $\left\{c_{i}: i=1,2, \ldots, n\right\} \subset V\left(R_{n}\right)$ and another belong to $\left\{d_{i}: i=1,2, \ldots, n\right\} \subset V\left(R_{n}\right)$. Without loss of generality we suppose that one resolving vertex is $c_{1}$ and the other is $d_{t},(1 \leq t \leq k+1)$. For $1 \leq t \leq k-1 \quad$ we have $r\left(a_{n} \mid\left\{c_{1}, d_{t}\right\}\right)=r\left(b_{n} \mid\left\{c_{1}, d_{t}\right\}\right)=(2, t+2) \quad$ and $\quad$ for $t=k \quad$ is $r\left(a_{k} \mid\left\{c_{1}, d_{t}\right\}\right)=r\left(c_{k} \mid\left\{c_{1}, d_{t}\right\}\right)=(k+1,1)$, similarly for $t=k+1 \quad$ than $\quad$ we have $r\left(a_{k+1} \mid\left\{c_{1}, d_{t}\right\}\right)=r\left(c_{k+2} \mid\left\{c_{1}, d_{t}\right\}\right)=(k+1,1)$, a contradiction.

Hence, from above it follows that there is no resolving set with two vertices for $V\left(R_{n}\right)$ implying that $\operatorname{dim}\left(R_{n}\right) \geq 3$

Case (ii): $n=2 k+1, k \geq 3, k \in I N$. Consider $W=\left\{b_{1}, b_{2}, b_{k+2}\right\} \subset V\left(R_{n}\right)$. We show that $W$ is a resolving set for $V\left(R_{n}\right)$. For this we find the representations of vertices of $V\left(R_{n}\right) \backslash W$ with respect to $W$. The representations of the vertices are as follow;

$$
\begin{aligned}
& r\left(a_{i} \mid W\right)= \begin{cases}(1,1, k+1), & \text { for } i=1 ; \\
(i, i-1, k+2-i), & \text { for } 2 \leq i \leq k+1 ; \\
(2 k+2-i, 3+2 k-i, i-k-1), & \text { for } k+2 \leq i \leq n .\end{cases} \\
& r\left(b_{i} \mid W\right)= \begin{cases}(i-1, i-2,2+k-i), & \text { for } 3 \leq i \leq k+1 ; \\
(2 k-i+2,2 k+3-i, i-2-k), & k+3 \leq i \leq n .\end{cases} \\
& r\left(c_{i} \mid W\right)= \begin{cases}(1,2, k+1), & i=1 ; \\
(i, i-1, k+3-i), & 2 \leq i \leq k+1 ; \\
(k+1, k+1,1), & i=k+2 ; \\
(2 k+3-i, 2 k+4-i, i-1-k), & k+3 \leq i \leq n .\end{cases}
\end{aligned}
$$




$$
r\left(d_{i} \mid W\right)= \begin{cases}(2,2, k+2), & \text { for } i=1 \\ (i+1, i, k+3-i), & \text { for } 2 \leq i \leq k+1 \\ (2 k+3-i, 4+2 k-i, i-k), & \text { for } k+2 \leq i \leq n\end{cases}
$$

Proceeding on same line as in case(i) we observe that there are no two vertices having the same representations, implying that $\operatorname{dim}\left(R_{n}\right) \leq 3$.

Also as in case(1), it can be shown that there is no set $W^{\prime}$ with $\left|W^{\prime}=2\right|$, such that $W^{\prime}$ is a resolving set for $V\left(R_{n}\right)$ for $n \geq 6$ and $n$ is odd. Thus, $\operatorname{dim}\left(R_{n}\right) \geq 3$. Hence $\operatorname{dim}\left(R_{n}\right)=3$. From case(i) and case(ii) we get $\operatorname{dim}\left(R_{n}\right)=3$.

\section{Conclusion}

In this paper we have studied the metric dimension of two families of graphs which are the extension of the antiprism graph. We have seen that the metric dimension of these graphs is finite and does not depend on the order of the graph and only three vertices appropriately chosen suffice to resolve all the vertices of these graphs.

\section{References}

[1] J. Caceres, C. Hernando, M. Mora, I. M. Pelayo, M. L. Puertas, C. Seara, D. R. Wood, On the metric dimension of cartesian product of graphs, SIAM J. Disc. Math. 2 (2007) 423-441.

[2] G. Chartrand, L. Eroh, M. A. Johnson, O. R. Oellermann, Resolvability in graphs and metric dimension of a graph, Disc. Appl. Math. 105 (2000) 99-113.

[3] F. Harary, R. A. Melter, On the metric dimension of a graph, Ars Combin. 2 (1976) 191-195.

[4] M. Imran, A. Q. Baig, A. Ahmad, Families of plane graphs with constant metric dimension, to appear in Utilitas Math.

[5] M. Imran, S. A. Bokhary, A. Q. Baig, On families of convex polytopes with constant metric dimension, Comput. Math. Appl. 60 (2010) 2629-2638.

[6] M. Imran, A. Q. Baig, M. K. Shafiq, Andrea Fenovcikova, Classes of convex polytopes with constant metric dimension, Utilitas, Math., in press

[7] I. Javaid, M. T. Rahim, K. Ali, Families of regular graphs with constant metric dimension, Utilitas Math. 75 (2008) 21-33.

[8] K. Karliraj, J. V. Vernold, On equatable coloring of helm and gear graphs, International J. Math. Combin., 4 (2010) 32-37.

[9] R. A. Melter, I. Tomescu, Metric bases in digital geometry, Computer Vision, Graphics, and Image Processing, 25 (1984) 113-121.

[10] P. J. Slater, Leaves of trees, Congress. Numer. 14 (1975) 549-559.

[11] P. J. Slater, Dominating and reference sets in graphs, J. Math. Phys. Sci. 22 (1998) 445-455. 